\title{
Philosophiques
}

\section{De Thomas More à Condorcet : une relève du discours utopique?}

\section{Charles Coutel}

Volume 24, numéro 2, automne 1997

URI : https://id.erudit.org/iderudit/027450ar

DOI : https://doi.org/10.7202/027450ar

Aller au sommaire du numéro

Éditeur(s)

Société de philosophie du Québec

ISSN

0316-2923 (imprimé)

1492-1391 (numérique)

Découvrir la revue

Citer cet article

Coutel, C. (1997). De Thomas More à Condorcet : une relève du discours utopique? Philosophiques, 24(2), 277-284. https://doi.org/10.7202/027450ar
Résumé de l'article

Cet article examine la relation complexe que Condorcet entretient avec l'idéal de perfectibilité hérité de la Philosophie des Lumières. L'Atlantide condorcétienne reprend et développe l'idéal utopique de T. More ; l'Europe est l'horizon de cette reprise critique de l'utopie, avec Condorcet l'espoir utopique s'accomplit dans une définition de l'Europe comme tâche éthique et politique. Le slogan actuel de la « fin des utopies "nous paraît incompatible avec la volonté de « construire l'Europe ". d'utilisation que vous pouvez consulter en ligne.

https://apropos.erudit.org/fr/usagers/politique-dutilisation/ 


\title{
DE THOMAS MORE À CONDORCET : UNE RELĖVE DU DISCOURS UTOPIOUE ?*
}

\author{
PAR \\ Charles Coutel
}

RÉSUMÉ : Cet article examine la relation complexe que Condorcet entretient avec l'idéal de perfectibilité hérité de la Philosophie des Lumières. L'Atlantide condorcétienne reprend et développe l'idéal utopique de T. More; l'Europe est l'horizon de cette reprise critique de l'utopie, avec Condorcet l'espoir utopique s'accomplit dans une définition de l'Europe comme tâche éthique et politique. Le slogan actuel de la "fin des utopies" nous paraît incompatible avec la volonté de " construire l'Europe ".

ABSTRACT: This article examines Condorcet's complex relationship with the ideal of perfectibility inherited from Enlightenment philosophy. Condorcet's Atlantis continues and develops More's utopian ideal; the horizon for this critical return to Utopia is Europe. In Condorcet the utopian hope is fulfilled in a definition of Europe as an ethical and political task. The present slogan of the " end of Utopias" seems to us to be incompatible with the will to "build Europe".

Comment le discours utopique est-il apparu? Cette question nous semble capitale en un temps qui ne cesse de proclamer la " fin des utopies". Le discour's utopique peut, certes, se clore mais à condition que l'espérance philosophique et politique dont il est porteur soit reconduite et comprise. La confrontation de T. More et de Condorcet peut aider à ce travail et à cette prise de conscience. En effet, l'étude de l'Utopie puis de l'Esquisse indique les conditions d'apparition, mais aussi de clôture du discours utopique. L'utopie de T. More est d'abord un livre qui s'organise autour de son impossibilité même (u-topie) et il nous semble que Condorcet, notamment dans le Fragment sur l'Atlantide qui suit l'Esquisse, dénoue cette impossibilité en l'immanentisant au sein d'une histoire générale de la liberté humaine. Cette hypothèse nous est apparue lorsque les commentateurs de l'Atlantide condorcétienne (K. M. Baker en 1975 
ou E. Brian en $1994^{1}$ ), intégrèrent précipitamment ce texte de 1793 1794 dans le " genre utopique » : ce jugement invite à l'analyse et à la méditation philosophique.

L'Atlantide condorcétienne est, à bien des égards, une problématisation du discours utopique, mais en même temps elle accomplit le projet de $\mathrm{T}$. More en nous parlant de la même espérance philosophique ; il s'agit ici de donner un lieu philosophique à cette espérance.

Condorcet problématise le discours utopique pour deux raisons : tout d'abord, il parvient à dépasser une des principales contradictions de T. More, formulée en 1969 par P. Mesnard ${ }^{2}$ : la société présentée par T. More est d'emblée parfaite, mais il n'est pas dit comment elle l'est devenue. La société utopienne, par là même, ne progresse pas. Or, Condorcet, par sa théorie de la perfectibilité, indique comment, en s'appuyant sur ses erreurs passées, toute société humaine peut progresser. Le discours utopique ne peut exister qu'en opérant un saut hors du présent, mais en même temps il perd d'un côté ce qu'il gagne de l'autre: l'audace théorique est contemporaine de l'impossibilité pratique; ce « double-lien méthodologique "est dénoué par Condorcet dans l'Esquisse. Mais ce dépassement n'abolit pas le projet utopique initial, il l'accomplit en lui assignant un nouveau lieu qu'il s'agit ici d'indiquer. Ce lieu recouvert par T. More et découvert par Condorcet est aussi une énigme pour nous aujourd'hui : si l'utopie n'a de lieu réel, elle a sans doute une raison d'être.

D'autre part, Condorcet nous aide à dépasser une autre difficulté majeure du discours utopique : comment décrire un monde qui n'existe pas et où le situer? On sait que T. More comme F. Bacon tournent le problème en recourant à la fiction d'un long voyage (qui rappelle l'expédition vers l'Amérique). La lecture attentive du texte de T. More révèle combien cette société future et rêvée emprunte, à son insu peut-être, des éléments au présent et aux cultures récemment découvertes (en Amérique) : les Utopiens ont de curieuses allures d'Indiens déguisés en Romains ! Condorcet, en revanche, ne pense pas l'avenir en ces termes, car il cherche à produire du nouveau en combinant les éléments du présent et non en les juxtaposant comme T. More.

Pour penser ces contradictions et les dépasser, il nous faut opérer un premier détour par le projet de T. More lui-même et le relire à partir de sa possible conclusion condorcétienne.

1. K. M. Baker, Condorcet, Raison et Politique, trad, Paris, Hermann, 1988, et E. Brian, La mesure de l'État, Paris, Albin Michel, 1994.

2. P. Mesnard, L'essor de la philosophie politique au XVI e siècle, Paris, Vrin, 1969, p. 175 ss. 


\section{Court détour par I. More}

Dans sa présentation de la revue La Bibliothèque de l'homme public, en 1791, Condorcet recommande chaudement la lecture de T. More et de F. Bacon, manifestant ainsi son intérêt pour le discours utopique $^{3}$. La référence explicite à l'Atlantide de F. Bacon, lui-même lecteur de T. More, indique plus qu'une filiation, elle situe une espérance commune : penser les contradictions d'un monde présent dans une projection idéale future. Mais T. More juxtapose les institutions utopiennes (dans la deuxième partie de son texte) comme il juxtapose la première et la deuxième partie de son ouvrage. Cette série de juxtapositions assigne au discours utopique ses limites et son originalité : ne faut-il pas s'arracher du présent pour penser un avenir meilleur? Cette force initiale devient aussitôt faiblesse comme nous le notions précédemment : comment réaliser ce qui s'avoue irréalisable ${ }^{4}$ ?' Que recouvre cette impossibilité même ? Pour répondre à cette question, il convient de lire T. More de plus près. Dans son texte $^{5}, \mathrm{~T}$. More propose une bien étrange construction philoso phique et rhétorique : Raphaël, le narrateur, aurait accompagné Amerigo Vespucci dans trois de ses voyages. T. More signale que son héros connaissait le grec et le latin et a s'était voué à l'étude de la philosophie $^{6}$ ». La première partie du texte s'achève par un récit décrivant un naufrage " près de l'île d'Utopie ${ }^{7}$; l'auteur indique qu'autrefois : "Quelques Romains, quelques Égyptiens furent jetés sur le rivage (de l'île d'Utopie); ils restèrent dans le pays ». Curieux pays où ces hommes des antipodes auraient accueilli si facilement la culture des Romains, des Grecs et des Égyptiens : l'utopie n'est-elle pas une tentative de penser ensemble l'antipode et l'antérieur? Tout se passe comme si $\mathrm{T}$. More voulait nous dire que le monde présent pourrait mieux penser ses contradictions s'il faisait l'effort de se laisser juger par un regard venant des antipodes (celui des Indiens, récemment découverts et dont beaucoup "fantasment "déjà l'innocence) et des mondes antérieurs (l’Égypte, la Grèce et

3. Nous renvoyons sur ce point à notre article, " La perfectibilité chez Condorcet " paru dans la revue Les Cahiers philosophiques en mars $1990, \mathbf{n}^{\circ} 42$, et à notre intervention dans le Colloque Condorcet de novembre 1994, "Utopie et perfectibilité : significations de l'Atlantide chez Condorcet "(Actes à paraître dans la revue Theoria de l'ENS Fontenay-Saint-Cloud). Dans notre ouvrage Politique de Condorcet, Paris, Payot, 1996, aux pages 217 ss, nous analysons la portée politique et la genèse épistémologique et institutionnelle de ce thème de l'Atlantide chez Condorcet.

4. Cette série de contradictions du discours utopique provoquerait une " pathologie "spécifique lors de toute tentative de a réalisation » effective des espérances utopiques, selon P. Watlawick, J. Weakland et R. Fisch dans le chapitre 6, intitulé "Le syndrome d'Utopie ", de leur ouvrage Changements, paradoxes et psychothérapie, Paris, Seuil, 1975, p. 66-81.

5. Voir T. More, L'Utopie, Paris, Garnier-Flammarion, 1987, p. 86, 132, 133.

6. Ibid., p. 86 .

7. Ibid., p. 132. 
Rome); mais cet effort semble si ardu que seul un arrachement hors du présent peut le rendre possible : les Utopiens sont déguisés à la fois en Indiens et en Romains, mais semblent ne pas avoir de visage.

Il faut rappeler que le texte de $\mathrm{T}$. More est à resituer dans un contexte historique qui connaît à la fois la redécouverte des textes de l'Antiquité gréco-latine et la découverte du Nouveau Monde ${ }^{8}$. Le discours utopique est une tentative synthétique pour " tenir ensemble " ces deux événements historiques, mais il est encore de l'ordre de la parataxe (juxtaposition des réformes dans l'île d'Utopie et juxtaposition des deux parties dı texte de T. More); Condorcet apporterait-il la syntaxe de cette parataxe initiale? Nous le pensons. Comme T. More, il relève l'importance et la convergence des deux événements historiques (redécouverte de l'Antiquité et découverte du Nouveau Monde) ${ }^{9}$; lui aussi veut les penser ensemble mais sur un autre mode que le discours utopique, tout en gardant l'intuition matinale et subversive de T. More. Le détour par l'antipode et l'antérieur possibilise le présent en le confrontant à sa propre perfectibilité. Le discours utopique de T. More est une première tentative individuelle pour juger un monde présent à partir du point de vue le plus éloigné dans l'espace (l'antipode) et le plus éloigné dans le temps (l'antérieur), mais il nous semble qu'un maillon manque encore. Ainsi, la monarchie anglaise et les effets du capitalisme sont jugés dans leur injustice initiale ; l'utopie confronte le présent à cette double altérité spatiotemporelle; mais cette confrontation jugée nécessaire ne parvient pas à se situer elle-même (u-topie). Cette abstraction sera critiquée par Machiavel, Spinoza puis Marx sur le mode du rejet radical de l'utopie. Or, on semble s'être peu intéressé à l'approche condorcétienne de l'utopie : Condorcet faisant partie des... utopistes, on ne cherchera pas chez lui ce qui complique et problématise le discours utopique. Pareil jugement devient plus grave si l'on décrète... la « fin " des utopies! Or, la critique de l'utopie n'est pas nécessairement son rejet, mais sa transcroissance raisonnée au sein d'une conception mélioriste de l'histoire ${ }^{10}$; c'est tout l'intérêt de l'Esquisse et du Fragment sur l'Atlantide de Condorcet. Ce philosophe des Lumières perçoit que T. More, par son geste philosophique inaugural (l'utopie), crée du possible dans un monde qui « n'en peut plus "; mais il sent aussi que ce discours ne peut penser ni sa propre genèse ni sa réalisation historique effective (lui qui analyse sans cesse les limites de

8. Cette volonté d'élargir et de problématiser le cadre philosophique de la pensée et de l'action grâce à la redécouverte de l'Antiquité et à la découverte du Nouveau Monde traverse aussi l'œuvre de Montaigne et de La Boétie.

9. Voir Condorcet, Esquisse d'un tableau historique des progrès de l'esprit humain (édition A. Pons), Paris, Garnier-Flammarion, 1988, p. 191-193.

10. Le méliorisme est la position philosophique qui considère que le monde peut être rendu meilleur par les initiatives et les efforts humains convenablement raisonnés et dirigés. Le méliorisme permet de ne pas avoir à choisir entre le pessimisme et l'optimisme. 
l'imitation des modèles politiques antiques par ses contemporains). L'Esquisse s'efforce d'intégrer dans le devenir humain les moments de la prise de conscience de $T$. More; la question devient alors : comment insérer dans l'histoire effective des hommes les exigences éthiques et politiques manifestées par l'espérance utopique? Ce qui donnerait un lieu... à l'utopie. Une nouvelle question surgit : quel cadre philosophique d'ensemble permettrait d'articuler le point de vue de l'antipode et le point de vue de l'antérieur comme instances critiques du présent ? La réponse à cette question est d'importance, car elle engage notre temps qui, proclamant la " fin " des utopies, risque d'oublier les formidables attentes philosophiques dont T. More serait le hérault humaniste initial.

Chez Condorcet, la prise en compte de l'antipode et de l'antérieur, dans la perspective d'un Montesquieu, s'unifie dans le projet récapitulatif de l'Atlantide qui doit autant à Platon qu'à Bacon ; l'Atlantide est projective, mais elle est aussi commémorative comme le projet encyclopédique lui-même qui combine sans cesse l'invention et l'inventaire. La théorie condorcétienne du progrès des Lumières parvient à historiciser ce que $T$. More ne faisait que proclamer; $\mathrm{T}$. More en reste à la protestation individuelle face à une société cruelle et à un pouvoir politique arbitraire. Il lui manque un cadre d'ensemble dont Condorcet va dessiner les contours dans l'Esquisse. La perfectibilité condorcétienne, héritière de Rousseau, fait glisser le " possible utopique "du possible possibilisé vers le possible possibilisant : l'avenir est pensé à partir des erreurs du passé et des contradictions du présent (ce que fait en partie T. More) mais aussi des potentialités intellectuelles, scientifiques et techniques contenues dans le présent. Condorcet met fin à a l'exil utopique » en proposant de dresser à la fin de l'Esquisse le "tableau de nos espérances". Condorcet critique bien l'abstraction du discours utopique, mais ne jette pas "le bébé avec l'eau du bain "et conserve la colère révolutionnaire de T. More, en l'insérant dans l'histoire. Cette immanentisation de l'espérance utopique n'est en aucune façon une réduction déterministe ou un fatalisme idolâtre du Progrès (ce que le XIX" siècle se plaît à voir chez Condorcet). Cette clôture de l'utopie est une ouverture et un appel à la responsabilité philosophique et politique. Mais pour mesurer la portée de cette redéfinition condorcétienne de l'utopie, un nouveau détour est requis : il nous donnera le moyen de comprendre comment Condorcet reprend et dépasse les contradictions initiales de T. More.

\section{Un nouveau détour : où il est question de l'Europe}

En 1992, R. Brague rédige un ouvrage consacré à l'idée d'Europe et organisé autour de l'hypothèse suivante qui pourrait nous donner une clef pour penser le lien philosophique entre $T$. More et Condorcet: l'Europe n'est pas une culture, mais un lieu de 
confrontation et d'échanges infinis entre les cultures qui nous viennent du passé (et singulièrement de Rome et de la Grèce) et les cultures qui viennent du plus lointain ; de l'antérieur et de l'antipode. L'Europe est cet échange et cette interlocution ; elle est transmission ${ }^{11}$. Être Européen, c'est pouvoir et vouloir conserver ce qui vient du passé et accueillir ce qui nous vient des antipodes et toute " renaissance „ européenne se caractériserait par cet effort pour se laisser juger par ces deux instances critiques : ce serait le cas au XVIII ${ }^{e}$ siècle avec des philosophes des Lumières comme Montesquieu, Diderot et Condorcet, mais aussi au XVI" siècle avec T. More. Cette première Renaissance se situe justement au moment précis de cetle double expérience de la redécouverte de l'Antiquité et de la découverte du Nouveau Monde. R. Brague ${ }^{12}$ nomme secondarité culturelle ce principe d'ouverture vers l'antérieur el l'antipode dans la définition de soi (ce geste est particulièrement évident chez Montesquieu, auteur des Lettres persanes et des Considérations sur la grandeur et la décadence des Romains). L'Europe est ce lieu de la confrontation permanente de l'antérieur et de l'antiporle dans la recherche de soi et de la vérité. Cette ouverture à l'altérité rendrait l'esprit européen vigilant et attentif à toute régression vers la barbarie (définie comme oubli des autres hommes et du passé). L'Europe est ouverture à autre que soi. R. Brague achève ainsi sa définition ${ }^{13}$ : "L'Europe se distingue des autres mondes culturels par le mode particulier de son rapport au propre : l'appropriation de ce qui est perçu comme étranger "

Par sa volonté de penser l'antipode et l'antérieur, le discours utopique de T. More nous dirait quelque chose sur la genèse de liidée politique et philosophique d'Europe: on saisit ce qu'a de contradictoire la double proclamation de la " nécessaire construction de l'Europe "et de... " l'actuelle fin des utopies". Il nous semble que T. More, par son récit utopique, indique en ne sachant où le situer (utopie), un lieu civilisationnel qui saurait penser la criticue du présent à partir d'une rationalité projetée dans l'avenir. Le discours utopique de T. More serait l'idée d'Europe se cherchant. Mais chez T. More, l'Europe aspire à elle-même en tant qu'horizon encore vague et aspiration impossible : espoir caressé de voir le monde présent se remettre globalement en cause en se situant imaginairement dans un monde plus ample qui intégrerait les leçons des Anciens et des autres cultures lointaines : ainsi, les Utopiens lisent les auteurs grecs et, précise T. More ${ }^{14}$ : "S’ils s'approprient si rapidement cette littérature (grecque) c'est, je pense, en vertu d'une certaine parenté " (souligné par

11. Voir R. Brague, Europe, la voie romaine, Paris, Criterion, 1992, p. 36. Nous développons cette problématique dans notre article "L'iclée d'Europe chez Condorcet " figurant dans notre ouvrage À l'école de Condorcet, contre l'orléanisme des esprits, Paris, Ellipses, 1996, p. 117-126.

12. Ibid, p. 116.

13. Ibid., p. 119.

14. T. More, L'Utopie, p. 186. 
nous). Ce geste narratif de T. More cristallise l'espoir de l'Europe humaniste du XVI ${ }^{\mathrm{e}}$ siècle ; il en manifeste aussi les limites.

En effet, dans le même temps, le récit de T. More trahit une impossibilité à pouvoir penser l'effectuation de cette idée d'Europe, comme le confirme l'insularité généralisée de la configuration utopique. L'idée d'Europe est ici figée aussitôt que revendiquée; ce qui fragilise le projet utopique dans une abstraction vite dénoncée par les critiques, comme nous le notions auparavant.

Or, il nous semble que l'idée d'Europe chez Condorcet, qui se développe à travers l'Esquisse et le Fragment sur l'Atlantide, permet une historicisation critique et heuristique du projet initial de T. More; c'est ce que nous avons nommé la relève du discours utopique. II convient de revenir à notre hypothèse de départ et d'examiner pourquoi l'Atlantide condorcétienne est l'idée d'Europe, éclairée par sa propre perfectibilité.

\section{L'Europe, l'Utopie, l'Atlantide}

L'Esquisse puis le Fragment sur l'Atlantide s'organisent autour d'un espoir: la connaissance précise des erreurs du passé et des découvertes scientifiques et techniques peut aider à libérer l'humanité en développant sa raison ; avec le même présupposé que Rousseau (l'homme est perfectible), Condorcet tire une conclusion inverse: les Lumières, orientées par la connaissance du passé, l'extension de l'imprimerie, l'instruction publique et une constitution républicaine indiqueront le chemin à l'humanité. L'Esquisse est, comme le suggère Condorcet lui-même, une " histoire de la liberté humaine ${ }^{15}$ ". Plus précisément encore, Condorcet rapproche l'invention de l'imprimerie... des deux événements majeurs que T. More prend lui aussi en compte dans son projet : la redécouverte de l'Antiquité et la découverte de l'Amérique ${ }^{16}$ et, précise Condorcet, " $[. .$.$] on vit naître en Europe une sorte de liberté de$ penser ${ }^{17}$ n. T. More est à l'exact point de recoupement de ces trois faits (L'Utopie date de 1516). L'imprimerie notamment (le projet de T. More est d'abord un livre) résume et magnifie le principe de secondarité culturelle défini auparavant: l'antipode et l'antérieur nous sont connus par les textes du passé et les récits de voyage; l'idée d'Europe a les contours d'une bibliothèque; d'où l'acharnement des ennemis des Lumières à empêcher le peuple de lire et à brûler les livres. Le livre rend co-présents l'antipode et l'antérieur au cœur d'une même exigence critique : lire est l'opération requise pour que la décentration vers l'antipode rejoigne la décentration vers l'antérieur. L'Europe est cette interlocution entre l'antériorité temporelle et cette altérité spatiale et culturelle, et il nous

15. Voir Condorcet, L'Esquisse, p. 79, 187-191.

16. Ibid., p. 191.

17. Ibid., p. 199. 
semble que cette intuition traverse le projet de T. More mais trouve son achèvement chez Condorcet. L'Europe des Lumières dont l'Atlantide condorcétienne est la figure critique et idéale reprend, en l'historicisant, l'intention utopique en lui assignant cette fois un lieu philosophique, politique et civilisationnel précis. Le concept condorcétien d'Europe serait ainsi le projet utopique intégré dans une genèse historique et critique (à la confluence des événements historiques majeurs au début du $X V I^{e}$ siècle), mais cette lecture historique n'est pas réductrice, car elle intègre l'espérance utopique dans le devenir perfectible de l'humanité tout entière. Par l'Atlantide, Condorcet se donne le moyen de faire de l'avenir une instance historique mais aussi infiniment libératrice. L'utopie devient une tâche et non un simple rêse; elle s'intègre dans une nécessaire poétique des Lumières.

Nos différents détours autorisent peut-être un réexamen final de notre perplexité initiale : T. More, par l'audace de sa pensée inaugure un genre admirable que les hommes ont méconnu et amoindri au point de confondre l'utopie avec l'ensemble des contresens commis sur elle. T. More a tenté d'écouter les voix du passé et celles des lointains, car il pressentait que pour être soi il convient de sortir de soi pour revenir à soi, ce qui est l'exigence même de la vie et de la pensée. Le cadre de cette aventure est l'Europe comme idée intégratrice de ce qui n'est pas elle. Cette identité pérégrinale est à l'wuvre dans le voyage vers l'Utopie qui est sans doute aussi irnportant que la présentation des institutions utopiennes. L'essentiel de cet héritage philosophique et politique passe dans l'Esquisse et le Fragment sur l'Atlantide, véritable testament des Lumières. L'Europe des Lumières serait-elle l'idée d'utopie consciente d'ellemême? T. More ou Condorcet ne nous sont ici d'aucun secours, car nous sommes aujourd'hui la réponse fragile à cette question inquiète.

Faculté de droit Alexis de Tocqueville

Universitéd'Artois 